\title{
La formation continue d'infirmières de soins à domicile : retombées perçues au regard de l'amélioration de la qualité des soins aux aînés.
}

Jérôme Ouellet

Université de Montréal, jerome.ouellet@umontreal.ca

Joséphine Mukamurera

Université de Sherbrooke, josephine.mukamurera@usherbrooke.ca

Follow this and additional works at: https://qane-afi.casn.ca/journal

Part of the Other Nursing Commons

\section{Recommended Citation}

Ouellet, Jérôme and Mukamurera, Joséphine (2015) "La formation continue d'infirmières de soins à domicile : retombées perçues au regard de l'amélioration de la qualité des soins aux aînés.," Quality Advancement in Nursing Education - Avancées en formation infirmière: Vol. 1: Iss. 3, Article 6.

DOI: https://doi.org/10.17483/2368-6669.1051

This Article is brought to you for free and open access by Quality Advancement in Nursing Education - Avancées en formation infirmière. It has been accepted for inclusion in Quality Advancement in Nursing Education - Avancées en formation infirmière by an authorized editor of Quality Advancement in Nursing Education - Avancées en formation infirmière. 


\section{La formation continue d'infirmières de soins à domicile : retombées perçues au}

regard de l'amélioration de la qualité des soins aux aînés.

\section{Cover Page Footnote}

La réalisation de cette recherche a été rendue possible grâce à une bourse d'études supérieures de deuxième cycle du Conseil de recherche en sciences humaines du Canada (CRSH). Merci particulier à Madame Joséphine Mukamurera pour sa contribution à la direction de cette recherche. 


\section{Introduction}

Le vieillissement de la population ainsi que la hausse des maladies chroniques, qui affectent les personnes âgées (Organisation mondiale de la santé [OMS], 2012), forcent les collectivités à intensifier le virage des soins vers la communauté entrepris depuis plus de vingt ans. Pour les aînés atteints de maladies chroniques et en perte d'autonomie, les soins à domicile (SAD) deviennent leur principal moyen d'accès aux services de santé dans leur milieu de vie (Infirmières et infirmiers en santé communautaire du Canada [IISCC], 2010). Dans ce virage des soins vers les collectivités, dont en $\mathrm{SAD}$, l'État québécois a confié aux infirmières une importante responsabilité dans la prise en charge des soins auparavant dispensés dans les hôpitaux. Toutefois, ce virage pose de nombreux défis aux infirmières de SAD. Elles doivent gérer des situations de soins complexes qui exigent la prise de décisions cliniques importantes, le plus souvent, sans le soutien direct d'autres professionnels (Deshaies, Bilodeau, et Leprohon, 2010). Elles doivent avoir de multiples connaissances, les mettre à jour continuellement et développer des compétences dans des domaines variés afin de prodiguer des soins de qualité. Or, au cours des dernières années et encore aujourd'hui, nous assistons de plus en plus à des coupures marquées dans le financement de la formation continue (FC) (Roussel, 2010). Les employeurs, y compris en santé, questionnent les retombées des activités de formation continue (AFC) suivies sur l'amélioration des pratiques.

Dans l'étude que nous avons réalisée, nous nous sommes intéressés au rapport qu'entretiennent des infirmières de SAD au regard de leur FC. Entre autres, nous avons exploré les retombées perçues des AFC que suivent les infirmières au regard de l'amélioration de la qualité des soins. Ainsi, le présent article met en lumière des retombées concrètes d'AFC que suivent les infirmières de $\mathrm{SAD}$, sur la qualité des soins qu'elles prodiguent. Avant de brosser le portrait de ces résultats, nous présentons la problématique ainsi que les principaux éléments du cadre conceptuel et de la méthodologie. Ensuite, nous mettrons en évidence les résultats sur les retombées des AFC en relation avec des expériences professionnelles et des exemples authentiques de la pratique clinique d'infirmières en SAD. Une discussion de ces résultats s'en suivra ainsi qu'une conclusion qui ouvrira des avenues de recherche et d'innovation en matière de FC.

\section{Problématique de la recherche}

Selon l'Organisation mondiale de la santé (OMS) (2010, p. 5), « la population âgée de 60 ans et plus a doublé depuis les années 1980 et devrait atteindre deux milliards d'ici $2050 »$. Les maladies chroniques telles le cancer, les problèmes cardiovasculaires, le diabète et les affections respiratoires touchent souvent les personnes âgées (OMS, 2012). Ainsi, le vieillissement de la population conjugué à la perte d'autonomie et aux maladies chroniques et comorbidités qui lui sont souvent associées forcent les collectivités à revoir les modes de prestations de soins pour les aînés. Leurs besoins de soins exercent d'importantes pressions sur les ressources en santé et constituent un défi en termes d'accessibilité afin de préserver leur qualité de vie à un coût socialement acceptable. L'un des moyens choisis par plusieurs des sociétés pour répondre à ces besoins grandissant des aînés a été le virage des soins vers la communauté, dont en SAD, en positionnant l'infirmière comme un acteur central. Non seulement les aînés vivent-ils plus longtemps qu'auparavant, mais ils vivent aussi plus souvent avec des situations de soins complexes (Levett-Jones, 2005). Les soins prodigués à domicile par les infirmières exigent un large éventail de connaissances ainsi que des compétences particulières. Parmi leurs responsabilités, on retrouve notamment : la gestion de la douleur et d'autres symptômes en soins 
palliatifs; l'évaluation du client atteint d'insuffisance cardiaque ou rénale dialysé à domicile; l'évaluation et la détermination du plan de traitements de plaies aigües ou chroniques; l'évaluation et le suivi de personnes sous antibiothérapie ou chimiothérapie intraveineuses; le suivi de personnes ventilées mécaniquement; l'évaluation de l'état mental et des fonctions cognitives chez la personne atteinte d'un déficit cognitif ou l'ajustement de l'insuline chez le patient diabétique (Ouellet, 2012). Ces responsabilités s'ajoutent à celles qui étaient déjà reconnues aux infirmières en santé communautaire : promouvoir la santé et prévenir la maladie. L'Association canadienne des soins et services à domicile (2008) qualifie le SAD de service essentiel. La pratique infirmière en SAD comporte son lot de défis et de particularités. Les infirmières doivent assurer les soins aux clientèles auparavant hospitalisées le plus souvent sans le soutien direct d'autres professionnels (Deshaies et al., 2010). Conséquemment, elles doivent faire preuve d'autonomie professionnelle et utiliser leur jugement clinique à bon escient dans leur évaluation de la santé et dans leurs interventions. Elles prennent des décisions cliniques qui ont des répercussions sur l'état de santé du client et sa famille et cela est reconnu depuis longtemps (Deshaies et al., 2010; Daley et Miller, 1996; Meyer, 1997). La pratique infirmière en $\mathrm{SAD}$ se distingue donc de celle qui est réalisée en milieu hospitalier. Les infirmières sont appelées à prodiguer des soins dans différents milieux dans lesquels elles doivent régulièrement résoudre des problèmes complexes, parfois même sans contact direct avec le client (Deshaies et al., 2010).

Considérant la vitesse à laquelle évoluent les savoirs, les infirmières qui œuvrent en SAD sont appelées à assumer des responsabilités pour lesquelles elles n'ont pas nécessairement été formées initialement (Lehoux et Law, 2004). En ce sens, la FC constitue un moyen privilégié pour poursuivre le développement des compétences nécessaires au suivi des personnes âgées dans la collectivité. Déjà, en 1990, l'OMS soulignait l'importance de la FC des travailleurs de la santé comme le pont entre la formation initiale et l'évolution scientifique et technologique (Abbatt et Mejia, 1990). Du côté des instances québécoises, les ministères de la Santé et de l'Éducation ainsi que l'Ordre des infirmières et infirmiers du Québec soutiennent l'importance de la FC pour assurer à la population des soins de qualité, y compris dans un contexte de SAD (Deshaies et al., 2010). Or, actuellement, peu d'études ont identifié ou évalué clairement les retombées à long terme des AFC que suivent les infirmières sur l'amélioration de la qualité des soins (Griscti et Jacono, 2006). Même si les instances dans le domaine de la santé soulignent l'importance de la FC, il n'en demeure pas moins que dans un contexte de pénurie de ressources et de restructuration comme c'est le cas actuellement, la FC est souvent la première cible visée et atteinte par les compressions budgétaires (Blythe, Baumann, O’Brien-Pallas, et Butt, 2003), ce qui complique l'accès à la $\mathrm{FC}$ pour les infirmières. Le Conference Board du Canada souligne, qu'au cours des dix dernières années, nous avons assisté à des coupures de l'ordre de $40 \%$ dans les budgets consacrés à la FC (Roussel, 2010). Ces coupures seraient attribuables au manque de preuves scientifiques qui attestent des retombées de la FC sur l'amélioration des pratiques. Or, lorsque nous avons recensé les écrits sur ce sujet, nous avons relevé des limites importantes quant aux méthodes des études qui remettent en question le potentiel des AFC de produire des retombées qui perdurent. En sciences infirmières, la plupart des études réalisées n'ont pas tenu compte de la pertinence des AFC suivies au regard de la pratique clinique, ce qui peut expliquer cette «absence de retombées ». Ces études présentent peu d'informations quant aux méthodes pédagogiques choisies pour réaliser les $\mathrm{AFC}$ qui ont été développées à l'intention des infirmières. De plus, elles n'ont pas tenu compte d'autres facteurs tels le manque de ressources (Blythe et al., 2003), les conditions de travail, le soutien des gestionnaires (Overton et MacVicar, 
2008), la motivation des infirmières (Beatty, 2000), sachant que ces facteurs peuvent limiter les retombées des AFC. Même si quelques études se sont, jusqu'à présent, intéressées aux enjeux organisationnels de la FC, à son efficacité ainsi qu'à d'autres facteurs qui peuvent en influencer son développement, son implantation et la participation des infirmières à cette dernière, à notre connaissance, une seule étude a été réalisée en SAD et elle remonte à 1996. Ainsi, l'étude que nous avons réalisée visait à définir le rapport à la $\mathrm{FC}$ d'infirmières en $\mathrm{SAD}$. Entre autres, nous nous sommes intéressés à identifier les retombées perçues des AFC que suivent les infirmières au regard de l'amélioration de la qualité des soins qu'elles prodiguent.

\section{Cadre conceptuel de la recherche}

La FC en sciences infirmières est un concept plutôt imprécis. Nous avons pu remonter jusqu'en 1978 pour retracer les premières définitions de la formation infirmière continue (FIC) (McNally, 1978). Au fil du temps, le concept de développement professionnel continu a peu à peu pris la place du concept de formation infirmière continue en l'englobant, mais sans s'y limiter (American Nurses Association [ANA], 1994; Lawton et Wimpenny; 2003; National Nursing Staff Development Organization et ANA, 2010). Dans le contexte qui nous concerne, nous avons décidé de définir la FC comme étant : « [...] un processus ininterrompu et permanent dans lequel l'infirmière s'engage activement tout au long de sa vie professionnelle à acquérir de nouvelles connaissances qui lui permettront de développer ses compétences par des activités d'apprentissage qui répondent à des besoins personnels ou professionnels » (Ouellet, 2012, p. 63). Dans le présent article, nous considérons que les expressions « formation continue (FC) » et «formation infirmière continue (FIC)» sont des synonymes et qu'elles peuvent être interchangeables. Pour réaliser cette recherche, nous nous sommes principalement basés sur les modèles proposés par Cervero (1985), Hegge (1985) et l'ANA (1994) comme cadre conceptuel pour la recherche. Ces trois modèles établissent des liens entre la FC des infirmières et l'amélioration de la pratique clinique ainsi que, ultimement, le rehaussement de la qualité de soins au client. Brièvement, le modèle de Hegge (1985) présente une approche réflexive et circulaire de la FIC qui comprend l'identification des besoins d'apprentissage et des ressources, la planification et la réalisation des activités de FC, et, enfin, l'évaluation des retombées en fonction de la pratique clinique. Le modèle de Cervero (1985), lui, conçoit la FC des infirmières comme un changement social qui est proposé à l'infirmière pour ultimement améliorer la qualité des soins selon six dimensions en interaction: le système social, l'individu comme professionnel, l'offre des activités de FIC, le changement proposé, le changement de comportement et le résultat sur le client. Le modèle de Cervero (1985) s'intéresse au résultat pour le client, ce qui s'apparente aux « retombées dans la pratique » du modèle de Hegge (1985). Quant au modèle de l'ANA (1994), il attire l'attention sur la qualité, le coût et l'efficience du soin, les facteurs environnementaux, les caractéristiques de l'apprenant et les options éducationnelles. Le modèle de l'ANA présente des similitudes avec les deux précédents modèles. Il intègre la prise en compte de l'individu du modèle de Hegge (1985) par le biais des caractéristiques des apprenants; le système social du modèle de Cervero (1985) par le biais des facteurs environnementaux, ainsi que le transfert dans la pratique (Hegge, 1985) et le résultat sur le client (Cervero, 1985) par la qualité, le coût et l'efficience du soin. On peut donc dire que ces modèles, bien qu'ils aient des différences, se rejoignent dans leur finalité qui est l'amélioration continue de la qualité des soins infirmiers dispensés; ce qui est au cœur de notre recherche. En plus de ces trois modèles évoqués ci-haut, notre recherche s'appuie sur des écrits qui se sont intéressés à des facteurs qui peuvent influencer la FIC. Certains traitent, par exemple, des motifs psychologiques qui mènent les infirmières à suivre des AFC (Billet, 2008), du processus 
d'apprentissage ainsi que d'expériences personnelles et professionnelles (Hugues, 2005), alors que d'autres s'intéressent davantage à l'environnement professionnel (Bahn, 2007), au soutien de l'employeur (Overton et MacVicar, 2008), aux exigences professionnelles (Lehoux et Law, 2004) ainsi qu'aux besoins de la clientèle (Levett-Jones, 2005). L'ensemble des trois modèles et des recherches recensées nous ont servi de repères pour développer le guide d'entrevue et pour analyser les données.

\section{Méthodologie de la recherche}

La présente étude qualitative a été réalisée auprès de huit infirmières en SAD dans une ville de taille moyenne au Québec (Canada). L'échantillon de convenance comptait huit infirmières dont la formation initiale était de niveau collégial ou universitaire. Leur nombre d'années d'expérience variait entre trois et plus de 21 ans et leur nombre d'années d'expérience en SAD se situait entre un et 20 ans. Des entrevues individuelles semi-dirigées ont été réalisées auprès des huit infirmières volontaires et ont été transcrites intégralement au fur et à mesure, tout en prenant soin de retirer les données sensibles afin d'assurer la confidentialité et l'anonymat des participants à la recherche. Après six entrevues, nous avons noté une redondance dans les propos des infirmières concernant les thèmes traités dans cet article. Les huit entrevues ont tout de même été analysées. Les données collectées en 2011 et 2012 ont fait l'objet d'une analyse thématique (Paillé et Mucchielli, 2003). Certains principes découlant de l'analyse des données qualitatives de Miles et Huberman (2003) ont guidé notre démarche de réduction, de condensation et d'interprétation des données. Des moyens tels l'intracodage, l'intercodage (Miles et Huberman, 2003) et le retour aux acteurs - appelé aussi le contrôle par les acteurs (Mukamurera, Lacourse, et Couturier, 2006) ont permis de valider nos analyses et d'en assurer la fiabilité. Un taux de fiabilité de $95 \%$ et $92 \%$ a été obtenu respectivement pour l'intracodage et l'intercodage. Lors du retour aux acteurs, quatre des huit participantes à la recherche se sont prononcées sur la congruence entre les propos qu'elles avaient tenus et l'analyse qui en avait été faite. Deux d'entre elles ont aussi demandé à ce que certaines informations sensibles soient retirées de la présentation des résultats. Les quatre autres participantes à la recherche n'ont pas commenté les analyses qui leur ont été envoyées. Dans la section suivante, nous présentons les principaux résultats de la recherche à propos de la pertinence des AFC suivies et de leurs retombées dans la pratique infirmière en SAD.

\section{Résultats}

À partir de récits d'expériences et de FC formulés par des infirmières de SAD, nous avons découvert que les AFC qu'elles suivent peuvent, à certaines conditions, améliorer la qualité des soins prodigués aux patients et à leur famille. Dans la présente section, nous traiterons d'abord de la pertinence des AFC suivies par les infirmières ${ }^{1}$, qui est la principale condition, pour qu'elles puissent améliorer la qualité des soins. Ensuite, nous expliciterons certaines des retombées concrètes sur les soins que dispensent les infirmières en SAD.

\footnotetext{
${ }^{1}$ Pour alléger le texte de cette section, nous avons choisi de présenter la plupart des résultats en référant de manière générale à «l'infirmière » ou aux « infirmières ». Il faut cependant comprendre que les propos et les analyses proviennent de l'échantillon des infirmières qui ont participé à l'étude et ne visent pas l'ensemble des infirmières qui travaillent en SAD.
} 


\section{Pertinence des AFC suivies}

L'analyse des données a permis de voir que les infirmières choisissent des AFC en raison de leur pertinence au regard de leur pratique. La pertinence, pour les infirmières interviewées, réfère à l'utilité des activités suivies dans la pratique clinique. C'est l'utilité perçue qui oriente leurs choix d'AFC et leur participation à celles-ci. En corollaire à cela, elles affirment que la plupart des AFC auxquelles elles participent sont pertinentes à leur pratique clinique.

«[...] moi je vais aller chercher la formation continue qui va m'être directement utile parce que je n'ai pas beaucoup de temps. [...] Il faut que ça ait un sens dans ce que je fais, auprès de mes clients. La plupart du temps, les activités de formation continue que je suis sont pertinentes à ma pratique et répondent à mes besoins ».

Par ailleurs, selon l'ensemble des infirmières interviewées, les AFC qu'elles suivent et qu'elles jugent pertinentes à leur pratique contribuent à améliorer la qualité des soins qu'elles prodiguent et ce, de manière soutenue dans le temps, ce qui a été peu documenté dans les études jusqu'à ce jour. Cependant, nous avons aussi pu constater que la pertinence des AFC ne repose pas uniquement sur l'utilité perçue au plan de la pratique. Les infirmières considèrent aussi important le fait de fonder leurs interventions sur des sources reconnues, dont des résultats probants. Les propos tenus par les infirmières nous ont également permis d'identifier des caractéristiques qui définissent ce qu'elles considèrent comme étant des AFC pertinentes. Ainsi, une AFC est jugée pertinente lorsqu'elle :

- est développée par un professionnel (ex. : infirmière ou médecin) dont les connaissances, l'expertise et les compétences sont reconnues de la part des infirmières ;

- apporte de nouvelles connaissances, de nouveaux outils ou ressources à l'infirmière, dans le cadre de son travail (ex.: présentation de nouveaux services ou d'organismes communautaires) ;

- est directement en lien avec les besoins de santé de la clientèle dont l'infirmière assure le suivi à domicile;

- tient compte de la réalité de l'environnement de pratique des SAD;

- ne nécessite pas de démarches complexes (exemple d'une démarche complexe: l'approbation d'un nouveau formulaire) de la part de l'infirmière pour modifier ses pratiques;

- produit des résultats concrets et positifs auprès du client.

Ainsi, nous avons pu définir, selon les infirmières qui ont participé à la recherche, les principaux éléments qui caractérisent la pertinence d'une AFC, cette «pertinence » semblant être intimement liée aux retombées potentielles des AFC qu'elles suivent sur la qualité des soins prodigués en SAD.

\section{Retombées perçues des AFC des infirmières sur la qualité des soins aux aînés en SAD}

Même si ce ne sont pas toutes les AFC qui ont des retombées positives sur la qualité des soins, les infirmières qui ont participé à la recherche ont cependant souligné que la majorité des AFC qu'elles suivent contribuent à améliorer, même à long terme, la qualité des soins qu'elles prodiguent aux aînés. Ce sont ces retombées que nous souhaitions identifier dans le cadre de notre recherche. 
Nous avons pu relever que les AFC pertinentes que suivent les infirmières en SAD permettent d'améliorer la qualité des soins principalement à deux égards : le premier étant l'évaluation des problèmes et des besoins des patients et le second étant l'intervention infirmière auprès de cette même clientèle. Nous traiterons de ces deux dimensions.

Évaluation plus exhaustive des problèmes et des besoins des patients suivis en SAD. L'amélioration de la qualité de l'évaluation infirmière se caractérise par une évaluation plus exhaustive des problèmes et des besoins des patients à domicile qui sont suivis par l'infirmière. En effet, les AFC permettent à l'infirmière d'acquérir des connaissances qui contribuent à améliorer la qualité de l'évaluation du client parce qu'elles permettent à l'infirmière de comprendre plus en profondeur les problèmes et les besoins de santé des aînés $(n=5)$.

«[...] on se spécialise et la formation continue nous permet qu'on puisse pousser plus loin nos connaissances, les approfondir, pour devenir encore plus spécialisées. »

De plus, les infirmières croient que la FC les outille pour développer et surtout consolider leurs capacités évaluatives afin de mieux assurer le suivi des clients à domicile, sans que ceux-ci aient, par exemple, à se déplacer ou encore à séjourner à l'urgence lorsqu'ils présentent des situations de santé moins stables $(\mathrm{n}=8)$.

«[...] la FC, bien ça nous permet de pousser plus loin, de mieux comprendre la situation du patient et de répondre à son besoin plutôt que, par exemple, de l'envoyer à l'urgence. On conserve notre maintien à domicile. On atteint notre but. Tu sais, quand on évalue bien le patient et qu'on sait comment bien le faire, [...] le patient peut rester chez lui, souvent. »

Les connaissances acquises au moyen de la FC sont de différentes natures: physiopathologiques, pharmacologiques, biologiques, etc. Elles peuvent aussi être en lien avec des habiletés techniques comme lorsqu'il s'agit de réaliser l'examen physique (ex. : observation, auscultation, palpation et percussion). Dans certaines situations, l'évaluation de l'infirmière permet à cette dernière d'intervenir sur des problèmes qui ne sont pas nécessairement d'ordre physique ou mental, mais de nature plus «sociale » et, le cas échéant, de référer le patient à un service ou un professionnel plus approprié.

«En fait, on fait et on gère tout. Tu sais, quand le patient ne va pas bien, il appelle et c'est nous qui l'évaluons; faisons les démarches auprès des médecins, des pharmacies si les médicaments ne sont pas corrects. Les relocalisations aussi. Souvent on détecte des affaires, toutes sortes de situations, parfois des crises familiales, et c'est nous, après avoir évalué, qui décidons d'envoyer le patient à l'hôpital ou non ou de le référer à un autre professionnel. C'est un contexte de première ligne et il faut avoir de la formation [continue]. »

Cette évaluation plus exhaustive que réalise l'infirmière lui permet dans un premier temps de fonder ses décisions et orientations cliniques sur des justifications plus solides $(n=5)$ et, dans un deuxième temps, d'anticiper ou encore de détecter plus précocement des complications $(\mathrm{n}=4)$ ou d'assurer une prise en charge plus efficace des situations de santé dites «instables » $(n=8)$. Les paragraphes qui suivent expliciteront chacune de ces dimensions que nous venons d'énoncer.

Justification plus solide des décisions et des orientations cliniques. En réalisant une évaluation de la condition de santé du client qui est plus exhaustive, l'infirmière comprend 
davantage la situation de ce dernier, ce qui fait en sorte qu'elle peut mieux justifier ses orientations cliniques ou ses soins et traitements en fonction, par exemple, du plan de traitement médical $(n=5)$. Par conséquent, elle peut avoir un apport non négligeable aux décisions médicales qui concernent le client.

"Par exemple, je vais maintenant faire les indices tibio-brachiaux, quelque chose que je ne faisais pas avant. Je n'avais pas tendance à penser de faire ça. J'envoyais un fax au médecin et lui ne savait pas quoi faire avec ça. Maintenant, je peux faire un indice et je suis plus outillée pour ensuite parler au médecin. Je suis mieux outillée, par exemple, pour argumenter quand le patient devrait aller passer un doppler. Là, j'ai l'information qu'il faut et le médecin me fait plus confiance avec cette information-là. Je suis plus crédible. »

Au-delà d'orienter ses propres interventions de soins auprès du client, l'évaluation que réalise l'infirmière permet aussi à d'autres professionnels de mieux répondre aux besoins du client. Concrètement, l'évaluation de l'infirmière peut, par exemple, permettre au médecin de modifier le traitement insulinique d'un patient diabétique ou encore de permettre à l'ergothérapeute de déterminer les équipements nécessaires à l'adaptation de l'environnement domiciliaire du patient afin d'assurer sa sécurité (ex. : installation de barres d'appui). Par conséquent, plus l'infirmière est en mesure d'évaluer en profondeur la situation du client, plus les informations qu'elle transmet au médecin ou à un autre professionnel sont utiles à ce dernier pour orienter ses décisions cliniques. C'est ce que soutiennent les infirmières que nous avons interviewées. Finalement, des infirmières $(n=3)$ ont aussi souligné que le fait de réaliser une évaluation qui soit exhaustive leur assure une importante crédibilité professionnelle non seulement auprès des patients ou encore de collègues, mais aussi auprès des médecins et des autres professionnels. La FC reçue semble donc rehausser la qualité de l'évaluation de la situation du patient et permettre ainsi une prise en charge adéquate.

Anticipation et détection précoces des complications et prise en charge efficace des situations de santé instables. En plus de permettre de justifier plus solidement leurs décisions cliniques en comprenant mieux et plus en profondeur la situation de santé du client, l'évaluation plus exhaustive que réalisent les infirmières des problèmes ou des besoins de santé du client, dont elles assurent le suivi, leur permet également d'anticiper ou de détecter plus précocement et avec justesse les signes et symptômes d'une complication imminente ou d'une détérioration de la condition clinique du patient $(n=3)$. Les AFC que suivent les infirmières leur permettent de repousser leurs limites en termes de capacités évaluatives. Elles remarquent les retombées de ces $\mathrm{AFC}$ dans des circonstances où les personnes présentent des situations de santé instables. À titre d'exemple, les infirmières ont mentionné que suite à des AFC, elles détectent mieux lorsqu'un patient insuffisant cardiaque présente une surcharge pulmonaire, car elles sont en mesure d'observer chez ce dernier une distension des jugulaires et un gain pondéral associé à une rétention hydrique ou encore d'entendre des anomalies à l'auscultation. Il en va également de même dans l'identification du délirium chez les patients âgés :

"Je me souviendrai toujours de cette formation sur le délirium. C’était un colloque. Avec la clientèle en perte d'autonomie liée au vieillissement et en soins palliatifs, c'est utile. Je suis capable de déceler plus rapidement un délirium parce que j'en connais bien maintenant la symptomatologie. C'est un plus. Décelé tôt, traité tôt, donc moins de complications. C'est une application directe dans ma pratique. » 
Non seulement la FC permet-elle d'améliorer la qualité de l'évaluation infirmière comme nous venons de le présenter, mais les AFC que suivent les infirmières, lorsqu'elles sont pertinentes, peuvent aussi contribuer à améliorer la qualité de leurs interventions infirmières. Les paragraphes qui suivent traiteront de cet aspect.

Amélioration de la qualité des interventions infirmières. Pour les infirmières ayant participé à la recherche, les AFC suivies ont également des retombées sur les interventions thérapeutiques qu'elles réalisent auprès de la clientèle. C'est ce qu'ont rapporté les huit participantes. Ces retombées prennent différentes formes selon la situation de soins. Nous avons identifié cinq aspects des interventions infirmières pour lesquels la FC a contribué à leur amélioration directement auprès de la clientèle. Nous les présentons de façon plus détaillée.

Optimisation dans le choix du traitement. Parmi les cinq aspects que nous avons identifiés, quant à l'amélioration de la qualité de soins des infirmières suite aux AFC, le premier réfère à l'optimisation dans le choix du traitement pour un problème de santé donné qui requiert un suivi, parfois à long terme, de la part de l'infirmière en SAD.

"[Les retombées des AFC], oui je les vois, surtout en soins de plaies, parce que c'est nous qui décidons du plan de traitements. [...]. Je n'avais pas une grande formation en soins de plaies. Les cours que j'avais eus à l'université n'étaient pas encore ferrés. Ça a donc grandement amélioré ma pratique. C'est pour cette raison que je crois que la formation [continue], plus on en aura, plus nous irons loin dans nos évaluations. Ça va tellement aider la pratique. Un exemple, j'ai une de mes collègues qui est venue me consulter. Une de ses clientes était suivie depuis des années parce que ses jambes coulaient. Avec le traitement que nous avons appliqué après notre discussion avec la stomothérapeute, on a pu guérir la plaie en trois mois. C'est donc concluant. [...] Cette formation-là m'a amenée à pousser plus loin et surtout à trouver la raison de la cause [plaie qui ne guérissait pas] et aussi à mieux intervenir. »

Cet extrait d'entrevue montre qu'en plus de réaliser une meilleure évaluation de la condition de santé du client, les AFC suivies par l'infirmière lui permettent d'optimiser les traitements qu'elle choisit d'appliquer selon le problème identifié chez le client. Le choix du meilleur traitement possible par l'infirmière permettrait par conséquent d'accélérer le processus de guérison et peut-être dans certains cas, de réduire la durée ou l'intensité du suivi.

Précision mieux définie des balises et des limites de ses interventions. Les AFC que suit l'infirmière lui permettent aussi de préciser les limites ou encore les balises à partir desquelles elle est en mesure d'intervenir auprès du client qui présente une problématique particulière et ainsi de le référer à un autre professionnel, lorsque sa situation dépasse son expertise ou encore son champ d'exercice $(n=4)$.

«Si six mois plus tard [après l'activité de FC suivie], je vois tel diagnostic ou tel problème, là tu te dis que tu as vu ça quelque part et tu vas voir dans tes biblio [bibliographies] qui sont souvent données dans les conférences et là tu vas voir ces informations-là. Tu vas, tu sors le texte et bon il y a ça et ça. C'est quoi les balises de détresse du trouble d'adaptation? Ah ok, au niveau physiologique, les troubles d'adaptation, ça se manifeste de telle ou telle façon. Au niveau psychologique, c'est telle autre chose. Écoute, mon patient en a deux sur trois, oui, il y a un problème, un trouble d'adaptation. Donc pour moi, ça me permet de cerner et à partir du moment 
où je cerne une problématique, je me dis ok, à mon niveau, je suis encore capable d'intervenir et de travailler la problématique avec le client et sa famille. Mais quand à un moment donné tu vois que tous les facteurs sont tous là, je l'accompagne, mais je me rends bien compte que j'ai besoin de quelqu'un d'autre pour intervenir. »

Ainsi, les AFC suivies par les infirmières leur permettent non seulement de prodiguer les soins de qualité dans les limites de leur expertise, mais aussi de déterminer dans quelle mesure l'apport d'un autre professionnel est nécessaire pour répondre adéquatement au besoin du client.

Exhaustivité de l'enseignement et de l'information au patient. Certaines des infirmières nous ont aussi mentionné, pendant les entrevues, que les AFC qu'elles suivent leur permettent d'améliorer l'information qu'elles donnent et l'enseignement qu'elles réalisent auprès du client et de sa famille $(n=2)$.

«Tu sais, comme les néo pulmonaires, avec les différents stades de cancer, tu comprends mieux pourquoi à tel stade on donne encore de la chimiothérapie et pas à l'autre stade. Maintenant, comme je connais et que je comprends mieux les traitements que le patient reçoit, je peux mieux l'informer, lui enseigner ou encore répondre adéquatement à ses questions. »

L'infirmière met donc à profit les connaissances acquises par la $\mathrm{FC}$ dans sa relation thérapeutique avec le client. Ces activités de FC suivies lui permettent de donner plus d'informations au client sur sa situation de santé afin que celui-ci comprenne mieux les soins et les traitements qui lui sont prodigués et le suivi qui en découle.

Protection des droits, soutien et accompagnement accrus du client et de sa famille. Toutes les infirmières que nous avons interviewées ont mis en lumière l'importance de leur rôle quant à leur responsabilité professionnelle de protéger (et de défendre) les droits de leurs clients et de leur famille, de les soutenir et de les accompagner dans des périodes de vulnérabilité et de fragilité. Ainsi, dans ce genre de situations, les infirmières soutiennent que les AFC suivies leur permettent de mieux protéger, de soutenir et d'accompagner leurs clients et leur famille, donc de mieux jouer leur rôle à cet égard.

"J'ai pris aussi [la formation continue] sur la douleur. Ça c'était sur mon temps que j'y allais; me former sur l'évaluation et les types de douleurs, les sortes d'analgésie, les médecines alternatives, etc. On a de tout ça. Les gens magasinent et quelque part ça fait partie du processus. Je ne me demande pas de tout connaître, mais je me dois de donner la bonne information au client et de l'orienter vers la bonne ressource au besoin si moi je ne sais pas. Je vais essayer de trouver de l'information de mon côté aussi. C'est aussi une partie de mon travail d'advocacy de défendre les droits des patients et des familles que je suis comme infirmière [...] Parfois tu es informée par le médecin et là le patient et la famille qui vivent dans un contexte précaire veulent payer une fortune pour des injections "miracles». À ce moment-là, sans les décourager, les gens ont toujours besoin d'espoir, mais c'est aussi dans mon rôle de les protéger, de les soutenir et de les accompagner dans ce cheminement-là.

Comme en témoigne cet extrait, les AFC que suivent les infirmières leur permettent non seulement de donner des informations justes au client, mais aussi de voir à protéger ses intérêts, 
notamment dans des situations de vulnérabilité où le pronostic est généralement sombre à plus ou moins court terme.

Amélioration de la sécurité et meilleure gestion des risques. Les AFC semblent aussi contribuer à améliorer la sécurité des interventions infirmières à domicile et à mieux gérer les risques qui leur sont inhérents. Deux infirmières ont fait part de cet aspect. L'exemple le plus éloquent qui nous a été relaté concerne l'administration d'un produit de chimiothérapie.

"On avait eu une formation sur le Méthotrexate [un type de chimiothérapie]. Souvent on ne savait pas trop comment on devait vraiment le donner. Certains se blousaient d'autres se gantaient seulement. Donc là, après cette formation, on a une façon uniforme et pareille qu'on est supposé utiliser pour administrer le traitement. Les blouses, les gants et la petite poubelle. Avant, on utilisait la boîte jaune pour les aiguilles. Ce sont des soins plus sécuritaires pour les patients, mais aussi pour nous. »

Certaines AFC que suivent les infirmières permettent donc d'intervenir en toute sécurité dans le cadre des activités professionnelles qu'elles réalisent auprès des clients à domicile. Par conséquent, ces AFC peuvent à la fois contribuer à assurer la protection des clients, et, pour les infirmières, à répondre aux impératifs de santé et de sécurité au travail.

En résumé, l'analyse des données que nous avons recueillies auprès d'infirmières en $\mathrm{SAD}$, met en évidence des retombées que peuvent avoir les AFC dans les soins directs qu'elles prodiguent aux clients dont elles assurent le suivi clinique. Ces retombées se répercutent à deux niveaux : au regard de leur évaluation des besoins de leur clientèle ainsi qu'au regard de leurs interventions auprès de cette dernière. Ces retombées semblent aussi perdurer dans le temps, car bon nombre de témoignages que nous avons reçus faisaient référence à des AFC qui avaient parfois été suivies il y a de cela plus de cinq ans. La pertinence des AFC au regard de la pratique clinique apparaît comme la pierre angulaire, voire une condition sine qua none, pour des retombées positives et durables de ces AFC sur le travail quotidien des infirmières.

\section{Discussion}

L'analyse des retombées des AFC suivies par les infirmières en SAD soulève une question intéressante relativement au transfert des apprentissages de la FC à la pratique clinique. Rappelons qu'assez tôt dans les années 1990, nous retrouvions déjà cette préoccupation de documenter les effets des activités de FC que suivent les infirmières sur l'amélioration de la qualité des soins (Barriball, While, et Norman, 1992). D'ailleurs, les modèles théoriques que nous avons présentés dans le cadre conceptuel ont tous une dimension associée aux retombées des activités de FC. Pour Hegge (1985), il s'agit de l'évaluation de l'apprentissage en fonction de la pratique clinique; pour Cervero (1985), il s'agit du résultat sur le client et finalement pour l'ANA (1994), ces retombées des AFC se traduisent par la qualité, le coût et l'efficience du soin.

Or, les résultats des études quantitatives disponibles ne permettent pas d'établir de liens concrets entre les connaissances acquises dans une AFC et l'amélioration de la qualité des soins dans la pratique clinique. Là où notre recherche apporte un éclairage nouveau, c'est justement dans la façon d'évaluer les retombées des AFC sur la pratique clinique à partir de l'expérience concrète des infirmières. Nous avons ainsi relevé des retombées positives importantes et celles-ci touchent particulièrement deux aspects de la pratique clinique des infirmières : l'évaluation et l'intervention. Ces deux éléments associés à l'amélioration de la qualité des soins étaient aussi 
les cibles de l'étude quantitative réalisée au Québec par Boutin et al. (2006) chez des infirmières du service Info-Santé. Contrairement à la nôtre, cette recherche, qui mesurait les retombées d'AFC sur une période de neuf mois, apportait des bémols quant à l'effet à long terme des AFC.

Pour notre part, l'approche qualitative que nous avons retenue pour la réalisation de notre étude nous a permis de relever des situations concrètes de transfert durable des apprentissages de la $\mathrm{FC}$ et d'améliorations tangibles de la pratique clinique dans des cas spécifiques. Effectivement, dans certaines de nos entrevues, les infirmières ont fait référence à des AFC qu'elles avaient suivies il y a parfois deux à cinq ans et elles étaient en mesure de donner des exemples concrets de la façon dont ces connaissances, qu'elles avaient acquises par des AFC, étaient appliquées encore aujourd'hui dans leur pratique clinique. Les entrevues réalisées ont permis de comprendre comment les infirmières arrivaient à modifier leur pratique clinique et à y intégrer de nouvelles connaissances. L'exemple de l'infirmière qui est maintenant en mesure de détecter plus rapidement un œdème aigu du poumon chez un client insuffisant cardiaque parce qu'elle a développé une compétence en examen clinique illustre bien dans quelle mesure la FC peut contribuer de façon durable à l'amélioration de la qualité des soins. Dans le même sens, un impact positif de la FC suivie a été noté avec les formations en soins de plaies et le dépistage du délirium. Les infirmières sont en mesure de dire concrètement ce en quoi les activités qu'elles réalisent (ou qu'elles ont réalisées) contribuent à l'amélioration de la qualité des soins et ce, à moyen ou à long terme, lorsqu'elles sont en contact avec des situations qui les incitent à utiliser leurs nouvelles connaissances. Les retombées ne se limitent pas seulement au patient; elles représentent aussi des bénéfices pour le milieu clinique de soins, voire pour le système de santé et la société, élément qu'il est important de garder en tête dans le contexte récurrent de restrictions budgétaires. Une illustration de l'effet boule de neige des retombées positives de la FC est l'exemple du client qui reçoit, selon une ordonnance, un diurétique par voie intraveineuse à son domicile, puis fait l'objet d'un suivi qui sera adapté à sa condition. Cela signifie une visite de moins à l'urgence et dans bon nombre de cas, un épisode hospitalier de moins et parfois aussi un séjour de moins en soins aigus. Comme société, il apparaît clair que c'est un gain important et qu'il devient avantageux d'investir dans de telles AFC qui visent à améliorer les capacités évaluatives et d'intervention des infirmières en $\mathrm{SAD}$, voire des infirmières œuvrant dans d'autres secteurs cliniques.

Évidemment, l'offre de FC n'est pas une finalité en soi et n'implique pas à priori des retombées positives. Il importe, tout comme l'ont souligné les infirmières participant à la recherche, que les AFC rendues accessibles répondent minimalement aux problèmes des patients dont elles assurent le suivi à long terme ainsi qu'au besoin de développement professionnel de ces infirmières. C'est donc toute la question de la pertinence des AFC offertes qui doit être évaluée sérieusement avant d'engager une infirmière dans une activité quelconque de FC. C'est d'ailleurs la position que met de l'avant l'OMS (1990), position qui est toujours d'actualité :

«[...] la formation continue n'est satisfaisante que dans la mesure où elle s'attache à résoudre les vrais problèmes reconnus par la communauté, le système de santé et les praticiens de la santé. [...] L'évaluation des besoins de formation continue (et l'utilisation intelligente de cette évaluation) est peut-être la fonction la plus importante d'un système de formation continue » (Abbatt et Mejia, 1990, p. 24-27).

Il s'avère que l'évaluation des besoins de FC des infirmières est un incontournable et qu'elle doit être réalisée avec des mécanismes qui permettent de les identifier adéquatement afin d'orienter judicieusement les décideurs dans les AFC qu'ils rendent disponibles aux infirmières. 
Par ailleurs, considérant le potentiel des retombées lorsque les AFC offertes sont pertinentes à la pratique clinique, le financement consacré à la FC devrait être perçu davantage comme un investissement plutôt qu'une dépense, avec évidemment un suivi post-formation. En effet, comme le souligne Bourgeois (1991, p.20),

"Le développement des ressources humaines est de plus en plus considéré comme une variable stratégique de la performance des entreprises et des organisations. [...] La formation ne doit plus suivre [...], mais anticiper. Le fait de voir la formation [continue] de plus en plus comme un investissement pour l'organisation implique aussi qu'on attend davantage en retour, au même titre que n'importe quel autre investissement».

De plus, en s'engageant dans une approche réflexive (avant, pendant et après l'action) autour de la situation problématique de santé, démarche qui s'apparente assez bien à celle que propose Hegge (1985) dans son modèle d'apprentissage autodirigé, les infirmières en arrivent à identifier leurs besoins d'apprentissage et à évaluer les ressources, pour ensuite successivement planifier des activités d'apprentissage, les réaliser, réinvestir les acquis dans leur pratique clinique et finalement évaluer si le problème de départ est résolu. Ceci corrobore les résultats de l'étude de Walsh et de ses collaborateurs (2006) qui étayent que les infirmières qui réalisent des AFC qui s'inscrivent dans une approche réflexive (comme c'est le cas chez les infirmières en $\mathrm{SAD}$ que nous avons interviewées) interviennent plus efficacement auprès de la clientèle. Cette étude (Walsh et al., 2006) dans laquelle les infirmières avaient suivi une AFC, dont l'approche pédagogique était de type réflexif, a été en mesure de montrer que les infirmières assuraient un meilleur management de la fièvre en pédiatrie, notamment en évaluant et en traitant plus efficacement cette dernière. Ces auteurs soulignent par conséquent que les approches pédagogiques de type réflexif et actif doivent nécessairement être mises de l'avant pour mieux favoriser le transfert des connaissances théoriques à la pratique clinique. De telles approches favorisent la réflexion non seulement avant et pendant 1'action, mais aussi après celle-ci (Ibid., 2006). C'est de cette démarche réflexive dont nous ont fait part les infirmières dans le cadre des entrevues. Finalement, nos résultats et ceux de l'étude de Walsh et al. (2006) nous portent à penser que, si l'AFC que réalise l'infirmière a un sens pour elle en fonction de sa pratique clinique, cette même AFC aura plus de chance d'exercer un impact tangible sur l'efficacité de l'évaluation et de l'intervention de l'infirmière auprès de la clientèle.

\section{Conclusion}

De nature qualitative, notre recherche portant sur le rapport à la FC d'infirmières de SAD a permis d'apporter un éclairage plus approfondi et actuel, à partir de descriptions d'expériences et d'exemples authentiques, des retombées d'AFC qu'elles suivent de même que de leur pratique. Nous avons ainsi pu mettre en évidence que des AFC que suivent des infirmières en SAD peuvent avoir des retombées positives sur leur pratique clinique en contribuant à l'amélioration de leur évaluation et de leurs interventions auprès de la clientèle dans un contexte d'isolement professionnel où leur pratique requiert une autonomie accrue. Loin de prétendre d'avoir épuisé le sujet, nous croyons que la recherche présentée ne fait que paver la voie au développement d'autres études qui pourront être réalisées avec un échantillon plus important. Par ailleurs, il est possible que les infirmières qui se sont portées volontaires pour participer à notre recherche soient celles qui croyaient déjà en la valeur de la FC. Cela peut faire en sorte que seules les retombées positives soient mises en lumière. Néanmoins, comme nous l'avons déjà mentionné dans la problématique, le champ de la recherche s'est très peu intéressé aux 
infirmières en SAD, malgré un virage des soins de plus en plus prononcé vers la communauté. Cette étude-ci met en perspective l'importance de la FC dans un tel contexte. Ses résultats permettent aussi d'envisager quelques pistes de recommandations pour améliorer l'offre et la qualité de la FC en SAD et ainsi permettre d'en optimiser les retombées. Il s'agirait notamment, pour les infirmières, de documenter les besoins de la clientèle dont elles assurent le suivi, et, corollairement, d'établir leurs propres besoins de développement professionnel pour faire valoir, auprès de leur employeur, leurs demandes de FC. Des AFC ancrées dans la pratique quotidienne, des démarches de FC fondées sur la réflexion de même que des activités développées localement seraient à encourager dans la mesure où elles favorisent le transfert des connaissances dans la pratique. Il va sans dire qu'un suivi post-formation (Boutin et al., 2006) s'avère aussi nécessaire afin d'évaluer l'évolution des pratiques et de cerner les aspects tant professionnels qu'organisationnels et environnementaux favorables ou non au changement de pratique chez les infirmières. Finalement, il serait fortement souhaitable que les chercheurs, tant en sciences de la santé qu'en sciences de l'éducation, s'intéressent à développer des approches de recherche innovantes d'une part pour mieux documenter l'efficacité de la FC et d'autre part pour proposer des pistes d'amélioration tant cliniques que pédagogiques pour soutenir le transfert des connaissances à la pratique des infirmières. 


\section{Références}

Abbatt, F., et Mejia, A. (1990). La formation continue des personnels de santé-Manuel pour atelier. Genève: Organisation mondiale de la santé.

American Nurses Association. (1994). Standards for Nursing Professional Development: Continuing Education and Staff Development. Washington, DC: American Nurses Publishing.

Association canadienne de soins et services à domicile (2008). Les soins à domicile: le prochain service essentiel - Répondre aux besoins de notre population vieillissante. Repéré à http://www.cdnhomecare.ca/media.php?mid=1913

Bahn, D. (2007). Orientation of nurses towards formal and informal learning: Motives and perceptions. Nurse Education Today, 27, 723-730. http://dx.doi.org/10.1016/j.nedt.2006.10.006

Barriball, K., While, A., et Norman, I. (1992). Continuing professional education for qualified nurses: a review of the literature. Journal of Advanced Nursing, 17(9), 1129-1140. http://dx.doi.org/10.1111/j.1365-2648.1992.tb02048.x

Beatty, R. (2000). Rural nurses' attitudes toward participation in continuing professional education. Thèse de doctorat en éducation des adultes, Pennsylvania State University.

Billett, S. (2008). Learning throughout working life: A relational interdependence between personal and social agency. British Journal of Educational Studies, 56(1), 39-58. http://dx.doi.org/10.1111/j.1467-8527.2007.00394.x

Blythe, J., Baumann, A., O’Brien-Pallas, L., et Butt, M. (2003). La qualité de vie au travail et la valeur du travail infirmier. Dans C. Viens, M. Lavoie-Tremblay, et M. Mayrand Leclerc Optimisez votre environnement de travail en soins infirmiers (p. 23-36). Cap Rouge : Presses Inter Universitaires.

Bourgeois, É. (1991). L'analyse des besoins de formation dans les organisations : un modèle théorique et méthodologique. Mesure et évaluation en éducation, 14(1), 17-60.

Boutin, H., Robichaud, P., Valois, P., et Labrecque, M. (2006). Impact of a continuing Education Activity on the Quality of Telephone Interventions by Nurses in an Adult Asthma Client Base. Journal of Nursing Care Quality, 21(4), 335-343. http://dx.doi.org/10.1097/00001786-200610000-00011

Cervero, R. (1985). Continuing professional education and behavioral change: a model for research and evaluation. Journal of Continuing Education in Nursing, 16(3), 85-88.

Daley, B., et Miller, M. (1996). Defining home health care nursing: implications for continuing nursing education. Journal of Continuing Education In Nursing, 27(5), 228-237.

Deshaies, C., Bilodeau, H., et Leprohon, J. (2010). Lignes directrices : L'exercice infirmier en santé communautaire - soutien à domicile. Repéré sur le site de l'Ordre des infirmières et infirmiers du Québec : http://www.oiiq.org/sites/default/files/295LD-

Soutiendomicile_0.pdf 
Griscti, O., et Jacono, J. (2006). Effectiveness of continuing education programmes in nursing: literature review. Journal of Advanced Nursing, 55(4), 449-456. http://dx.doi.org/10.1111/j.1365-2648.2006.03940.x

Hegge, M. (1985). A model for Continuing Nursing Education Through Self-Directed Learning. Journal of Continuing Education in Nursing, 16(6), 205-213.

Hugues, E. (2005). Nurse's perceptions of continuing professional development. Nursing Standard, 19(43), 41-49.

Infirmières et infirmiers en santé communautaire du Canada (2010). Compétences en soins infirmiers à domicile. Repéré à www.chnc.ca/documents/CompetencesensoinsinfirmiersadomicileVersion1MArs2010.pdf

Lawton, S., et Wimpenny, P. (2003). Continuing professional development: a review. Nursing Standard, 26(17), 41-44.

Lehoux, P., et Law, S. (2004). Les technologies de soins à domicile : Enjeux de l'organisation et de la prestation des services du Québec. Repéré à http://www.associationhu.org/zdocpdf/20070715\%20SoinsA DomicileResume.pdf

Levett-Jones, T.-L. (2005). Continuing Education for Nurses : A Necessity or a Nicety? Continuing Education for Nurses, 36(5), 229-233.

McNally, J. (1978). A Resource Model for Continuing Education in Nursing. Journal of Continuing Education in Nursing, 9(4), 33-37.

Meyer, K. (1997). An educational program to prepare acute care nurses for a transition to home health care nursing. Journal of Continuing Education In Nursing, 28(3), 124.

Miles, M. B., et Huberman, M. A. (2003). Analyses des données qualitatives. (Trad. De M. Hlady Rispal révision scientifique de J. J. Bonniol), $2^{\mathrm{e}}$ édition. Paris : De Boeck.

Mukamurera, J., Lacourse, F., et Couturier, Y. (2006). Des avancées en analyse qualitative : pour une transparence et une systématisation des pratiques. Recherches qualitatives, 26(1), 110138.

National Nursing Staff Development Organization, et American Nurses Association. (2010). Nursing Professional Development - Scope and Standard of Practice, $2^{\mathrm{e}}$ édition. Silver Spring : Nursesbooks.org.

Organisation mondiale de la santé (2010). Plan d'action 2008-2013 pour la stratégie mondiale de lutte contre les maladies non transmissibles. Repéré à http://whqlibdoc.who.int/publications/2010/9789242597417_fre.pdf

Organisation mondiale de la santé (2012). Dix faits sur le vieillissement et la qualité de vie. Repéré à http://www.who.int/features/factfiles/ageing/fr/index.html

Ouellet, J. (2012). Le rapport à la formation infirmière continue chez des infirmières en soutien à domicile. Mémoire de maîtrise en sciences de l'éducation, Université de Sherbrooke, Sherbrooke, QC.

Overton, G., et MacVicar, R., (2008). Requesting a Commitment to Change: Conditions That Produce Behavioral or Attitudinal Commitment. Journal of Continuing Education in the Health Professions, 28(2), 60-66. http://dx.doi.org/10.1002/chp.158 
Paillé, P., et Mucchielli, A. (2003). L’analyse thématique. Dans P. Paillé et A. Mucchielli, L'analyse thématique en sciences humaines et sociales (p. 123-145). Paris: Armand Colin/VUEF.

Roussel, J.-F. (2010). L'apprentissage informel : une nouvelle donne, de nouveaux défis. Effectif 13(3), 14-19.

Santé et services sociaux Québec (2003). Pour faire les bons choix-Chez soi : le premier choix - La politique de soutien à domicile. Repréré à http://publications.msss.gouv.qc.ca/acrobat/f/documentation/ 2002/02-704-01.pdf

Walsh, A., Edwards, H., Courtney, M., Wilson, J., et Monaghan, S. (2006). Paediatric fever management: Continuing education for clinical nurses. Nurse Education Today, 26, 71-77. http://dx.doi.org/10.1016/j.nedt.2005.07.007 